\section{Fractal multiband antenna based on the Sierpinski gasket}

C. Puente, J. Romeu, R. Pous, X. Garcia and F. Benitez

Indexing terms: Antennas, Fractals

Experimental and computed results show a multiband behaviour over five bands for the new fractal Sierpinski antenna. Such a behaviour is based on the self-similarity properties of the antenna's fractal shape, which might open an alternative way for designing new multiband and frequency independent antennas.

Introduction: Most antenna designs are highly frequency dependent. The size of the antenna relative to the operating wavelength is the main bandwidth limiting factor. In the early 1960s, some selfscalable structures such as spirals, cones and log-periodic arrays were developed to design frequency independent antennas [2 - 5]. The common factor is that the shape of all these structures remains invariable under some scaling transformations.

Most fractal structures have a self-similar shape, which means that their shape is similar at different scales [6-8]. When we looked at the several parts that compose a fractal structure, a similar but smaller shape to the global object was found. An ideal fractal shape is said not to posses characteristic size, since an infinite number of scales and sizes are contained within the same object. Owing to this particular property of fractal structures, a multifrequency or frequency independent electromagnetic behaviour could be expected from a fractal body.

The interaction of some fractal structures with electromagnetic waves has been studied recently by Jaggard et al. [8 - 10]. Also, some fractal arrangements have been proposed for controlling the sidelobe ratio (SLR) in antenna arrays [9-11] and a first attempt to design multiband fractal arrays has recently been carried out [1]. In this Letter, the authors introduce a new fractal antenna based on the Sierpinski gasket which presents a self-similar behaviour at several bands.

Description: An equilateral triangular Sierpinski gasket conductor structure is the main body of the antenna. The antenna is printed over a thin dielectric substrate $\left(\varepsilon_{r}=2.17, h=0.127 \mathrm{~mm}\right)$ and mounted upside-down over a $80 \times 80 \mathrm{~cm}$ square conductor ground plane (Fig. 1). Such a monopole configuration is chosen because it greatly simplifies the antenna feeding scheme, which can be easily implemented by mounting a coaxial probe through the ground plane.

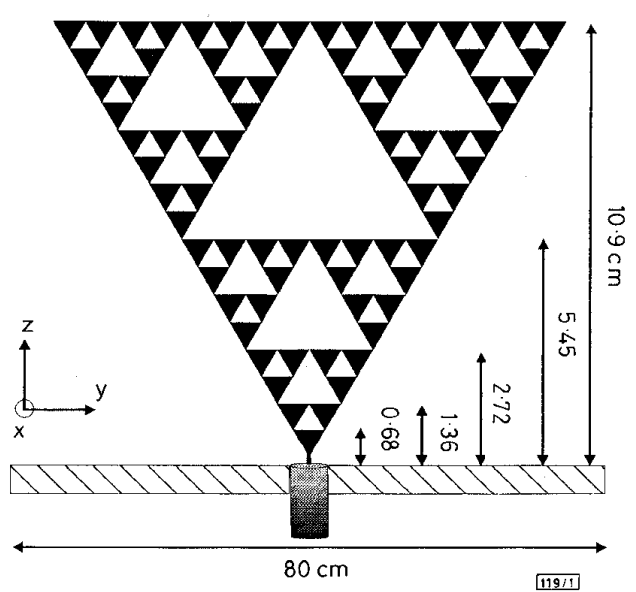

Fig. 1 Fractal Sierpinski monopole

Shape is self-similar at five different scales spaced by factor of 2

The fractal shape is constructed with five iterations. That is, the Sierpinski triangle appears at five different scales within the main structure. There is a factor of two between scales, which means that the Sierpinski triangle heights are $10.9,5.45,2.72,1.36$ and $0.68 \mathrm{~cm}$. This particular shape has been chosen owing to its similarity to the well known bow-tie (triangular) antenna. Since the triangular-like shape appears at five different scales, it could be expected that the Sierpinski antenna would behave similarly to a triangular antenna but at five different bands.
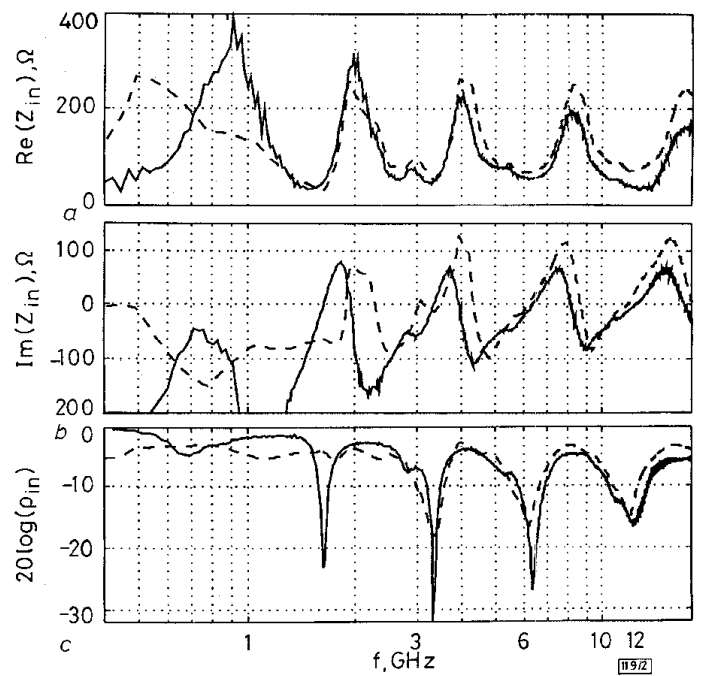

Fig. 2 Input impedance and input reflection coefficient

$a$ Real part, $b$ Imaginary part, $c$ Input impedance and input reflection coefficient

VSWR is minimum at $12.6,6.4,3.3,1.62$ and $0.64 \mathrm{GH} z$

- measured

- - - computed FDTD

Experimental and computed results: The antenna input reflection coefficient $\left(\rho_{i n}\right)$ relative to $50 \Omega$ was measured from $500 \mathrm{MHz}$ to $14 \mathrm{GHz}$ using an HP $8510 \mathrm{~B}$. Also, the antenna behaviour was simulated using the FDTD technique on a connection machine CM-5. The real and imaginary parts of the input impedance $\left(Z_{i j}\right)$ along with $\rho_{i n}$ are shown in Fig. 2. The measured parameters are shown in solid lines while the computed ones are shown in dashed lines.

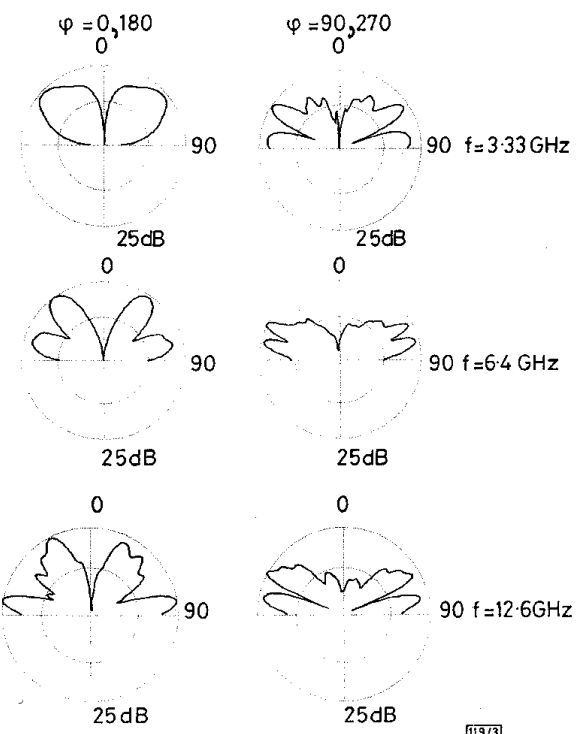

Fig. 3 Main cuts $\varphi=0^{\circ}, 180^{\circ} ; \varphi=90^{\circ}, 270^{\circ}$ of radiation pattern for $E_{\theta}$ component

Cuts were measured at three upper bands: $12.6,6.4$, and $3.3 \mathrm{GHz}$ and where normalised with respect to their maxima

All three plots clearly show a log-periodic behaviour, and the logperiod $(\delta)$ is $\sim$ a factor of two. In particular, the antenna is matched at five bands: $12.6,6.4,3.3,1.62$ and $0.64 \mathrm{GHz}$. While $\rho_{\text {in }}$ is below $-15 \mathrm{~dB}$ at the four upper bands, it is only $-5 \mathrm{~dB}$ at the lower one. Also, this band appears slightly shifted from its expected frequency position $(0.64 \mathrm{GHz}$ instead of $1.62 / \delta=$ $0.81 \mathrm{GHz}$ ). Both phenomena are also predicted by the numerical analysis and are most likely caused by the truncation of the fractal 
shape, which can not be implemented with an arbitrary number of scales and iterations. The truncation effect can also be related to the slight frequency shift and the worse matching of the upper band.

The complete radiation patterns were measured in an anechoic chamber at the lowest VSWR points of the three upper bands $(12.6,6.4$ and $3.3 \mathrm{GHz})$. The main cuts phiv $=0^{\circ}, 180^{\circ}, \varphi=90^{\circ}$, $270^{\circ}$ are shown in Figs. 3 and 4 for the $E_{\theta}$ and $E_{0}$ component of the electrical field respectively. All patterns are normalised with respect to their maxima and are represented in a polar form with a $25 \mathrm{~dB}$ dynamic range. Each cut presents a notable degree of similarity through the three bands, especially for the $E_{0}$ component which is the main component with an average of $30 \mathrm{~dB}$ above $E_{\mathrm{q}}$.
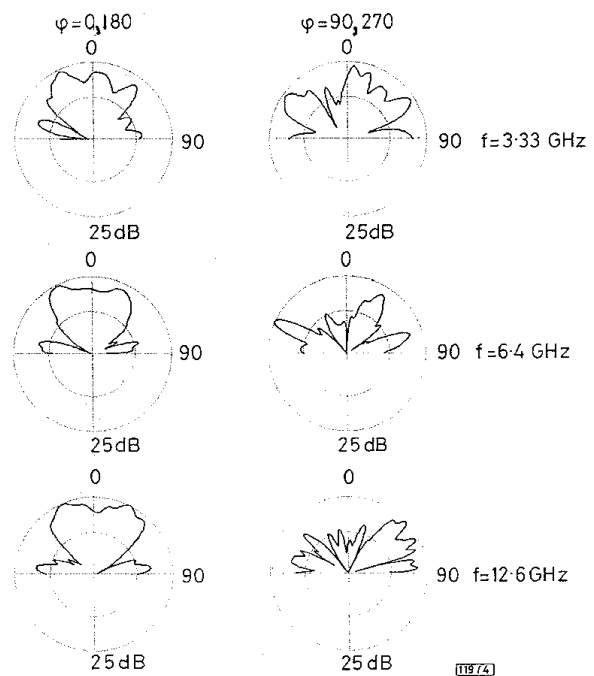

Fig. 4 Main cuts $\varphi=0^{\circ}, 180^{\circ} ; \varphi=90^{\circ}, 270^{\circ}$ of radiation pattern for $E_{\text {sp }}$ component

Cuts were measured at three upper bands: $12.6,6.4$, and $3.3 \mathrm{GHz}$ and where normalised with respect to their maxima. These maxima are, on average, $30 \mathrm{~dB}$ below same cuts for $E_{\theta}$ component.

Conclusions: Experimental and computed results on a novel fractal multiband antenna have been reported. The fractal antenna has shown a notable degree of similarity at five bands, the same number of scales over which the fractal structure appears similar. The bands are also spaced by a log-period of two, the same spacing that relates the five scales on the fractal shape. Thus, it can be concluded that the geometrical self-similarity properties of the fractal structure have been translated into its electromagnetic behaviour, and therefore, it seems that other fractal [12] shapes might help in the design of multiband or frequency independent antennas.

Acknowledgments: The authors would like to thank P. Mayes from the Electromagnetics Laboratory of the University of Mllinois for encouraging this work. They also thank L.L. Mila, A. Segui and $\mathrm{X}$. Fernàndez for contributions on the antenna measurements. This work has been financially supported by the 'Spanish Commission of Science and Technology' under grant TIC-93-0055C03-03.

(C) IEE 1996

Electronics Letters Online No: 19960033

10 October 1995

C. Puente, J. Romeu, R. Pous, X. Garcia and F. Benitez (Antenna Microwave, Radar and Photonics Group, Signal Theory and Gran Capità s/n, Mòdul D3, 08071 Barcelona, Spain)

\section{References}

1 PUENTE, C., and Pous, R.: 'Fractal design of multiband and low sidelobe arrays', IEEE Trans. Antennas Propag., 1994

2 MAYES, P.E., DESCHAMPS, G.A., and PATTON, W.T.: 'Backward-wave radiation from periodic structures and application to the design of frequency-indepentent antennas', Proc. IRE, 1961, 49, pp. 962-963

3 DYSON, J.D.: 'The equiangular spiral antenna', IRE Trans, 1959 AP-7, pp. 181-187
4 RUMSEY, V.H.: 'Frequency independent antennas' (Academic Press, New York, 1966)

5 CARREL, R.L.: 'Analysis and design of the log-periodic'. PhD Thesis, Department of Electrical Engineering, University of Illinois, Urbana-Champaign, USA, 1961

6 MANDELBROT, B.B.: 'The fractal geometry of nature' (W.H. Freeman and Company, San Francisco, 1983)

7 PEITGEN, H.O., JÜRGENS, H., and SAUPE, D.: 'Chaos and fractals' (Springer-Verlag, New York, 1992)

8 JAGGARD, D.L.: 'On fractal electrodynamics' in KRITIKOS, H.N., and JAGGARD, D.L. (Eds.): 'Recent advances in electromagnetic theory' (Springer-Verlag, London, 1990)

9 KIM, Y., and JAGGARD, D.L.: 'The fractal random array', Proc IEEE, 1986, 74, (9), pp. 1278-1280

10 JAGGARD, D.L., and SPIELMAN, T.: 'Triadic cantor target diffraction', Microw. Opt. Technol. Lett., 1992, 5, (9), pp. 460-466

11 GOUTELARD, C.: 'Fractal theory of large arrays of lacunar antennas'. Electromagnetic Wave Propagation Panel Symp. (AGARD-CP-528), 1992, pp. 35/1-15

12 Fractal and Multifractal antennas are patent pending

\section{Frequency agile slot-fed patch antenna}

\author{
G. Le Ray, M. Himdi and J.P. Daniel
}

Indexing terms: Patch antennas, Microstrip antennas

The authors present an original application of a slot-fed patch antenna. Voltage controlled tuning varactors are connected across the radiating edges of the patch antenna and the resonant frequency can be adjusted in a large frequency band (up to $1 \mathrm{GHz}$ ) while the impedance matching remains good. Results based on the transmission line model are compared with measurements.

Introduction: Microstrip patch antennas possess many desirable features, including light weight, low cost and conformability, which make them suitable for a wide variety of applications at microwave frequency. Unfortunately, microstrip antennas have a very low bandwidth characteristic. Previous work $[1,2]$ has shown that obtaining a tunable bandwidth up to $30 \%$ by incorporating a varactor diode into the patch antenna fed by a coaxial is possible. In this Letter, we present a tunable slot-fed patch antenna (Fig. 1), which keeps a good impedance matching for all the resonant frequencies ranging from 1.62 to $2.57 \mathrm{GHz}$.

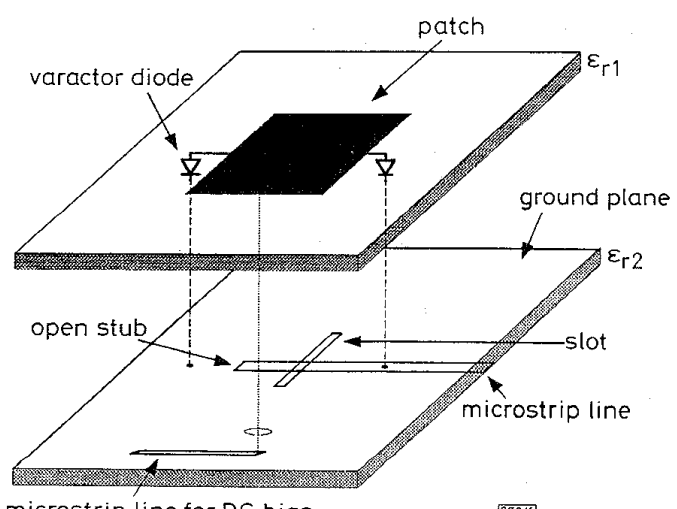

microstrip line for DC bias

Fig. 1 Slot fed patch antenna with varactors

Analysis: The slot-fed patch antenna has been analysed previously [3] using the transmission line model. This model has been used here because it is well suited for implementation on CAD existing circuit software (HP MDS for example). This type of structure also offers the advantage that the radiating microstrip patch is separated from the feed line by the ground plane (Fig. 1). It is possible to bias the active components incorporated into the patch antenna without large disturbance on feed line. For this application, varactor diodes are connected across the radiating edges of 